\title{
Dissociation between Private and Social Counterfactual Value Signals Following Ventromedial Prefrontal Cortex Damage
}

\author{
Nadège Bault ${ }^{1}$, Giuseppe di Pellegrino², Martina Puppi ${ }^{1}$, Gaëlle Opolczynski ${ }^{3}$, \\ Alessia Monti ${ }^{4}$, Davide Braghittoni ${ }^{2}$, Florence Thibaut $^{3}$, \\ Aldo Rustichini ${ }^{5}$, and Giorgio Coricelli ${ }^{1,6}$
}

\begin{abstract}
Individuals learn by comparing the outcome of chosen and unchosen actions. A negative counterfactual value signal is generated when this comparison is unfavorable. This can happen in private as well as in social settings-where the foregone outcome results from the choice of another person. We hypothesized that, despite sharing similar features such as supporting learning, these two counterfactual signals might implicate distinct brain networks. We conducted a neuropsychological study on the role of private and social counterfactual value signals in risky decision-making. Patients with lesions in the ventromedial prefrontal cortex (vmPFC), lesion controls, and healthy controls repeatedly chose between lotteries. In private trials, participants could observe the out-
\end{abstract}

\section{INTRODUCTION}

When we choose among alternatives, we may have the opportunity to compare the consequences of our choices with the consequences of foregone options or with the consequences of choices other people have made. In a private context, the unfavorable comparison between obtained and foregone outcomes (what might have been) can generate negative counterfactual value signals, called regret in economics (Bell, 1982; Loomes \& Sugden, 1982) and psychology (Zeelenberg, van Dijk, Manstead, \& van der Pligt, 2000), or fictive errors (Lohrenz, McCabe, Camerer, \& Montague, 2007) in neuroscience literature. In a social environment, unfavorable social comparison might generate interpersonal negative counterfactuals and thus elicit envy (Bault, Coricelli, \& Rustichini, 2008; Orthony, Clore, \& Collins, 1988). The two types of private and social counterfactual value signals may therefore be useful to improve our decisions.

\footnotetext{
${ }^{1}$ University of Trento, Mattarello, Italy, ${ }^{2}$ Centro Studi e Ricerche in Neuroscienze Cognitive, Cesena, Italy, ${ }^{3}$ Centre Hospitalier Universitaire de Rouen, ${ }^{4}$ Casa Cura Policlinico, Milan, Italy, ${ }^{5}$ University of Minnesota, ${ }^{6}$ University of Southern California
}

comes of their choices and the outcomes of the unselected lotteries. In social trials, participants could also see the other player's choices and outcome. At the time of outcome, vmPFC patients were insensitive to private counterfactual value signals, whereas their responses to social comparison were similar to those of control participants. At the time of choice, intact vmPFC was necessary to integrate counterfactual signals in decisions, although amelioration was observed during the course of the task, possibly driven by social trials. We conclude that if the vmPFC is critical in processing private counterfactual signals and in integrating those signals in decisionmaking, then distinct brain areas might support the processing of social counterfactual signals.
The involvement of the OFC in processing relative values and counterfactual signals is well established in private settings. Evidence from single-cell recordings in nonhuman primates suggests that the OFC carries information related to the relative value of rewarding options (Padoa-Schioppa \& Assad, 2006). OFC neurons responding to fictive value signals have been found in rats (Steiner \& Redish, 2014) and nonhuman primates (Abe \& Lee, 2011), influencing the animals' decisions toward options associated with hypothetical larger rewards in previous trials (Kim, Huh, Jang, Lee, \& Jung, 2015; Hayden, Pearson, \& Platt, 2009). In humans, the OFC encodes the difference between obtained and foregone outcomes (Boorman, Behrens, Woolrich, \& Rushworth, 2009; Coricelli et al., 2005), along with other brain areas such as the striatum and ACC (Lohrenz et al., 2007), and keeps track of the reward probability of the best unchosen option (Boorman, Behrens, \& Rushworth, 2011). Patients with lesions in ventromedial prefrontal cortex (vmPFC) — which includes the medial OFC—-failed to report regret after comparing the outcome of a bad choice with a better foregone outcome (Camille et al., 2004), although a recent study attributed this deficit to the lateral OFC (Levens et al., 2014). Moreover, vmPFC patients are 
impaired in anticipating the negative consequences of their choices, and they generally do not learn from negative experience (Wheeler \& Fellows, 2008).

By contrast, the implication of the OFC in valuating one's own reward relative to rewards obtained by others is unclear. Intracranial recordings in macaques in a social setting revealed that only a few neurons of the OFC respond to another's reward (Chang, Gariépy, \& Platt, 2013). Neurons of ACC, however, encoded either peers' rewards or both the monkey's own and its peers' rewards (Chang et al., 2013), which is consistent with a role of these neurons in social comparison. ACC neurons also predict another agent's decision to cooperate (Haroush \& Williams, 2015). In an fMRI study with humans (Bault, Joffily, Rustichini, \& Coricelli, 2011), we showed that the OFC activity was modulated by counterfactual comparisons, but OFC signals did not distinguish between situations where the counterfactual outcome was that of a nonchosen alternative or that of another participant. This distinction was, however, observed in the medial prefrontal cortex (mPFC; including parts of the ACC and the frontal polar cortex). The mPFC signaled events in which participants won more than their counterparts. Moreover, the mPFC was more activated during decisions made in a social than in a private context, concurring with the proposal that the ventrodorsal organization of the mPFC represents a distinction between self- versus other-oriented preferences (Sul et al., 2015; D’Argembeau et al., 2010; Mitchell, Macrae, \& Banaji, 2006). Nonetheless, other studies have reported activity in the OFC related to relative payoff in social comparison environments (Fliessbach et al., 2007) or to the prediction error of outcomes obtained by others (Burke, Tobler, Baddeley, \& Schultz, 2010). Thus, it is not clear whether the OFC is critical in encoding social comparison signals or whether more dorsal prefrontal areas might have a more important role.

Patients with lesions in the vmPFC are known to display abnormal emotional responses to reward and punishment (Bechara, Tranel, \& Damasio, 2000; Bechara, Tranel, Damasio, \& Damasio, 1996), as well as reduced self-conscious emotions such as shame or embarrassment (Beer, Heerey, Keltner, Scabini, \& Knight, 2003). vmPFC patients have also been reported to display inappropriate social behavior (Beer et al., 2003; Rolls, Hornak, Wade, \& McGrath, 1994; Stuss \& Benson, 1984). Evidence suggests they lack concern for social and moral rules and do not experience the aversive emotional responses to moral violations that are normally found in nonclinical populations ( $\mathrm{Gu}$ et al., 2015; Ciaramelli \& di Pellegrino, 2011; Koenigs et al., 2007). These observations have led to the argument that the impaired emotional system associated with vmPFC damage contributes to the patients' inappropriate social behavior (Kringelbach \& Rolls, 2004; Bechara, Damasio, \& Damasio, 2000; Elliott, Dolan, \& Frith, 2000). By contrast, in interactive games, vmPFC patients have been reported to display normal emotional reactions (Moretti, Dragone, \& di Pellegrino, 2008; Elliott et al., 2000), though their choice behavior was different from that of healthy participants and from patients with lesions in other parts of the brain. In the ultimatum game (Moretti et al., 2008; Koenigs \& Tranel, 2007), vmPFC patients rejected unfair offers more often than control participants, although showing normal levels of anger following unfair offers. Thus, the role of the vmPFC in processing social emotional responses and the nature of lesion patients' impairment in social behavior remains an open question. In particular, it is still unclear whether changes in social behavior following vmPFC lesions should be attributed to lesions in the OFC or rather to lesions in adjacent regions such as the frontal polar cortex and ACC (Rudebeck, Bannerman, \& Rushworth, 2008). We propose to investigate this question by directly comparing decisions in two environments that differ only in the dimension-social or nonsocial_of the counterfactual information available.

The aim of our study is twofold. First, we investigate the role of the OFC in the integration of social information in the decision-making process and specifically whether the OFC is necessary in the processing of social counterfactual value signals. Second, we seek to confirm the finding that vmPFC patients are impaired in the processing of private counterfactual value signals (Camille et al., 2004) by testing a different cohort of patients with brain lesion, as these results have been subject to debate (Levens et al., 2014). We suggest that the OFC might not be crucial in maintaining typical behavior in social settings, in contrast to private ones. Thus, patients with lesions in the vmPFC might still be able to use social information in their decisions. To test this hypothesis, we studied emotional and behavioral responses to private and social counterfactual comparison in patients with lesions affecting or sparing the prefrontal cortex (PFC) as well as in a group of healthy controls. Lastly, we investigated how these two signals affected subsequent choices.

\section{METHODS \\ Participants}

Twenty-five patients with brain lesion and 23 healthy participants took part in the study. We recruited 10 patients with lesions predominantly localized in the ventral part of the PFC (vmPFC group). The nonfrontal lesion comparison group included seven patients with lesions in parts of the occipital, parietal, or temporal cortex and sparing the frontal lobe. To perform voxel-based lesion-symptom mapping (VLSM) analyses, eight additional patients with prefrontal lesion, outside or not restricted to the vmPFC, were recruited.

Patients with brain damage were recruited from the Centre for Studies and Researches in Cognitive Neuroscience in Cesena (Italy), the Rouen Hospital Charles 
Table 1. Demographic Data of the Patients with PFC Lesion, Control Lesion Patients, and Healthy Control Participants

\begin{tabular}{|c|c|c|c|c|}
\hline Group & $\begin{array}{c}\text { Nationality } \\
\text { (French/Italian) }\end{array}$ & $\operatorname{Sex}(F / M)$ & $\begin{array}{l}\text { Age at Test } \\
\text { in Years (SD) }\end{array}$ & $\begin{array}{c}\text { Education in } \\
\text { Years (SD) }\end{array}$ \\
\hline vmPFC patients $(n=10)$ & $5 / 5$ & $1 / 9$ & $51(12)$ & $9(3)$ \\
\hline Other PFC patients $(n=8)$ & $1 / 7$ & $3 / 5$ & $54(13)$ & $12(1)$ \\
\hline Control lesion patients $(n=7)$ & $0 / 7$ & $5 / 2$ & $52(12)$ & $12(4)$ \\
\hline Healthy controls $(n=23)$ & $12 / 11$ & $4 / 19$ & $52(8)$ & $11(2)$ \\
\hline
\end{tabular}

Nicolle (France), and the Lyon Neurological Hospital (France). Potential participants suffering from visual, motor, or strong attention deficits that could interfere with their ability to perform the task were not included in the study. Twelve patients were taking psychoactive medications, most commonly anticonvulsants or antidepressants.

Control participants were recruited both in France and Italy via newspaper advertising. They were screened to exclude psychoactive medication and conditions of past or current psychological or neurological illness and history of head injury.

Patient and control groups differed neither in age (Table 1; Kruskal-Wallis $\chi^{2}=0.236, p=.97$ ) nor education level $\left(\chi^{2}=5.530, p=.14\right)$. When considered separately, neither patient group significantly differed from the healthy control group in age or level of education. All volunteers gave fully informed consent for the project, approved by the French National Ethical Committee (Comité Consultatif de Protection des Personnes dans la Recherche Biomédicale).

\section{Lesion Analysis}

All patients had stable, adult-onset, and well-defined lesions. Etiologies and lesion localization of all patients are described in Tables 2 and 3. Lesion analysis was based on the most recent clinical MRI $(n=16)$ or CT $(n=8)$. Lesions were manually drawn by an expert (blind to task performance) onto the normalized T1-weighted template MRI scan from the Montreal Neurological Institute (MNI) using MRIcron (Rorden, Karnath, \& Bonilha, 2007). Three-dimensional representations of lesions were created by drawing the lesion on the relevant slices of the template. When a surgical clip was present and artifacts made it difficult to observe damaged tissue, if damage was evident above and below the slices containing clip artifacts, then it was assumed that the lesion also included the region occupied by the clip. Volumes of lesions were computed with the VOI description tool of MRIcron. A prefrontal patient was included in the vmPFC group if the lesion met the following criteria: (1) more than 50\% of the lesion volume was situated in BA 10 and BA 11 and (2) the lesion's center of mass was situated in BA 11 or in the ventral part of BA 10. Therefore, patients with a lesion having a center of mass situated in the PFC who did not meet the vmPFC inclusion criteria were included only in the VLSM analyses. These patients had either nonfocal lesions of the vmPFC (extending to the lateral OFC and to other parts of PFC) or lesions damaging other parts of PFC. As they do not constitute a homogenous lesion group, we expected them to display different patterns of responses depending on the precise localization and extent of their lesions. The inclusion criteria for the lesion control group were a lesion's center of mass outside the frontal lobe and an intact prefrontal lobe. The overlap maps of lesions for the vmPFC and nonfrontal lesion comparison patient groups are shown in Figure 1.

\section{Neuropsychological Assessment}

A trained neuropsychologist administered a short neuropsychological test battery to all participants. The following tests were used to assess the participants' executive functions: the Trail-Making Test (Tombaugh, 2004; see Giovagnoli et al., 1996, for the Italian version without J and K), the Modified Card Sorting Test (Nelson, 1976), and the Frontal Assessment Battery (Dubois, Slachevsky, Litvan, \& Pillon, 2000; for Italian norms, see Appollonio et al., 2005). Scores for these tests are provided in Table 4.

\section{Experimental Procedure}

\section{Experimental Setup}

Lesion patients participated with a confederate of the same sex. The confederates were members of the research facility and unknown to the patients. They were instructed exactly as normal participants to ensure they would behave as such, but their data were later disregarded because of the possible decision bias induced by their knowledge of interacting with a patient. Healthy control participants were paired with another control of the same sex. One control participant played with a confederate because the second participant failed to show at the experimental session. The two participants of each pair sat in the same room and were separated by a panel wall. They were told they were about to play the same game together but that their payoff would only 
Table 2. Description of Patients' Lesions

\begin{tabular}{|c|c|c|c|}
\hline Patient & Etiology & Lesion Localization & Lesion Volume $\left(\mathrm{cm}^{3}\right)$ \\
\hline \multicolumn{4}{|c|}{ vmPFC Patients } \\
\hline 1 & Meningioma & vmPFC, bilateral & 31.38 \\
\hline 2 & Penetrating ballistic brain injury & vmPFC, bilateral & 19.73 \\
\hline 3 & Cranial trauma & Right vmPFC & 10.80 \\
\hline 4 & Angioma & Right vmPFC & 7.12 \\
\hline 5 & Meningioma & vmPFC, bilateral & 15.36 \\
\hline 6 & AcoA aneurysm & vmPFC, bilateral & 41.69 \\
\hline 7 & AcoA aneurysm & vmPFC, bilateral & 52.67 \\
\hline 8 & AcoA aneurysm & vmPFC, bilateral & 56.93 \\
\hline 9 & Cranial trauma & Left vmPFC, dlPFC & 27.55 \\
\hline 10 & ACoA aneurysm & Anterior OFC, ACC, frontopolar & 52.34 \\
\hline \multicolumn{4}{|c|}{ Other PFC Patients } \\
\hline 11 & Meningioma & Left vmPFC, temporal lobe & 57.63 \\
\hline 12 & AcoA aneurysm & Left vmPFC, left frontopolar, dlPFC & 95.69 \\
\hline 13 & $\begin{array}{l}\text { Aneurysm (right anterior } \\
\text { cerebral artery, A1-A2 tract) }\end{array}$ & ACC, subgenual area & 5.55 \\
\hline 14 & AcoA aneurysm & ACC & 25.91 \\
\hline 15 & Ischemic stroke & Left insula, dlPFC & 27.15 \\
\hline 16 & Ischemic stroke & Post. OFC, right dlPFC, temporal poles & 3.69 \\
\hline 17 & Meningioma & Right lateral OFC, ACC, frontopolar & 158.81 \\
\hline 18 & Ischemic stroke & Left insula, lateral PFC & 35.93 \\
\hline \multicolumn{4}{|c|}{ Control Lesion Patients } \\
\hline 19 & Glioblastoma & Right temporoparietal area & 127.83 \\
\hline 20 & Ischemic stroke & Right occipital cortex & 145.30 \\
\hline 21 & Venous stroke & Left middle temporal cortex & 56.14 \\
\hline 22 & Left ischemic stroke & Left insula, putamen Rolandic operculum & 25.63 \\
\hline 23 & Aneurysm (left medial cerebral artery) & Left middle temporal cortex, insula & 56.61 \\
\hline 24 & Spontaneous hemorrhage & Left occipital cortex & 7.25 \\
\hline 25 & Meningioma (postoperation) & Right occipital cortex & 13.35 \\
\hline
\end{tabular}

$\mathrm{AcoA}=$ anterior communicating artery; $\mathrm{dlPFC}=$ dorsolateral PFC.

depend on their own choices and not on the other's choices.

\section{Task}

A lottery task adapted from Bault et al. (2008) was used, manipulating the magnitude and probabilities of potential gains and losses (see Figure 2). Participants repeatedly chose between two lotteries, each with two possible outcomes from the set of values $\{-20 ;-5$; $+5 ;+20\}$. The probability of obtaining each outcome was taken from the set $\{0.2 ; 0.5 ; 0.8\}$. Probabilities were indicated by a sector on a circle. To make potential gains and losses easier to differentiate for patients, numbers and circle sectors were depicted in green for positive outcomes and in red for negative outcomes. The expected values of the two lotteries were always of the same sign (i.e., both positive or both negative). We ensured that the 
Table 3. Number of Voxels Lesioned in Each Brodmann's Area, Proportion of the Area with Lesions, and Maximum Overlap of Lesions, for the PFC Patients

\begin{tabular}{|c|c|c|c|c|c|c|}
\hline \multirow[b]{2}{*}{$B A$} & \multicolumn{3}{|c|}{ vmPFC Patients } & \multicolumn{3}{|c|}{ Other PFC Patients } \\
\hline & $\begin{array}{c}\text { No. of Voxels } \\
\text { with Lesion }\end{array}$ & $\begin{array}{l}\text { Proportion Area } \\
\text { with Lesion }\end{array}$ & $\begin{array}{l}\text { Maximum } \\
\text { Overlap }\end{array}$ & $\begin{array}{c}\text { No. of Voxels } \\
\text { with Lesion }\end{array}$ & $\begin{array}{l}\text { Proportion Area } \\
\text { with Lesion }\end{array}$ & $\begin{array}{c}\text { Maximum } \\
\text { Overlap }\end{array}$ \\
\hline 10 & 29138 & 0.783 & 7 & 33861 & 0.909 & 4 \\
\hline 11 & 50610 & 0.767 & 7 & 32941 & 0.499 & 3 \\
\hline 24 & 2206 & 0.21 & 4 & 4550 & 0.433 & 2 \\
\hline 25 & 702 & 0.051 & 4 & 3807 & 0.278 & 2 \\
\hline 32 & 11548 & 0.36 & 4 & 23340 & 0.728 & 2 \\
\hline 38 & 251 & 0.009 & 2 & 5935 & 0.219 & 3 \\
\hline 46 & 3778 & 0.132 & 3 & 24988 & 0.876 & 4 \\
\hline 47 & 8096 & 0.235 & 4 & 22996 & 0.667 & 3 \\
\hline 48 & 487 & 0.003 & 2 & 26805 & 0.169 & 2 \\
\hline 4 & - & - & - & 638 & 0.019 & 1 \\
\hline 6 & - & - & - & 11112 & 0.113 & 2 \\
\hline 8 & - & - & - & 14383 & 0.568 & 2 \\
\hline 9 & - & - & - & 29819 & 0.823 & 2 \\
\hline 43 & - & - & - & 96 & 0.014 & 1 \\
\hline 44 & - & - & - & 12305 & 0.653 & 2 \\
\hline 45 & - & - & - & 24119 & 0.846 & 3 \\
\hline
\end{tabular}

difference in expected values of the two lotteries of all pairs did not exceed $€ 7$ (see Bault et al., 2008, for a complete list of the lotteries pairs).

\section{Conditions}

In total, participants underwent 120 trials of the experimental task. The initial 80 trials had complete feedback, that is, the outcome of both lotteries was revealed, and could be either private ( 40 trials) or social ( 40 trials), in randomized order (see Figure 2). The following 40 trials had partial feedback, that is, only the outcome of the chosen lottery was revealed.

Private versus social trials (complete feedback) At the beginning of the trial, two lotteries were displayed, surrounded by a green-dotted square for private trials and a yellow-dotted square representing the second player in social trials. Participants could choose one of the two lotteries at any time by pressing the left or right arrow on the keyboard. In social trials, after making their choice, participants could also see the choice made by the second player. After a spinning period, the outcomes of both lotteries were displayed simultaneously. In private trials, participants could then compare their outcome to that of the unchosen lottery (counterfactual comparison). In social trials, they could compare their outcome to that of the second player (social comparison); they were told the lotteries were drawn once for the two computers, that the two players would see the same outcome screen, and that if both players had chosen the same lottery, they would receive the same payoff. Finally, in both private and social trials, participants were asked to provide a subjective emotional rating on the outcome of their choice ("How do you feel about the outcome of your choice?"), on a scale ranging from -50 (extremely negative) through 0 (neither positive nor negative) up to +50 (extremely positive).

Partial versus complete feedback We introduced partial feedback trials to ensure that the dissociation (amplification effect) between disappointment and regret, previously observed in healthy participants but not in vmPFC patients (Camille et al., 2004), was present with our participants. As it served replication purposes only, the partial feedback condition was always presented after the complete feedback condition. In this way, we avoided potential spillovers of the partial feedback condition on our conditions of interest (i.e., private vs. social with complete feedback). In partial feedback trials, the 
Figure 1. Overlap map of lesions of patients. (A) The overlap of lesions of the 10 vmPFC group are projected on seven axial slices of the ch2 template from the MRIcron software. (B) Overlap for the lesion control group. The scale represents the number of patients with overlapping lesions. The slices are oriented according to the neurological convention (i.e., left is left). The positions of the axial slices are displayed on the right bottom sagittal slice.

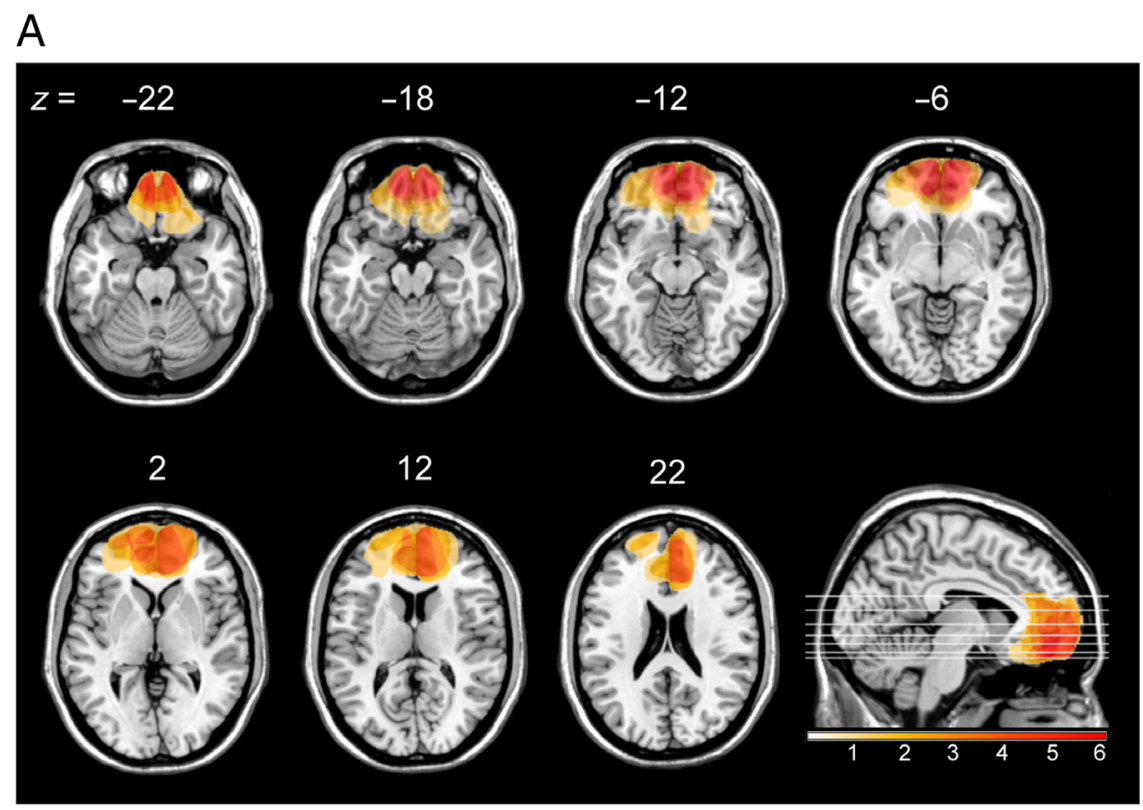

$\mathrm{B}$

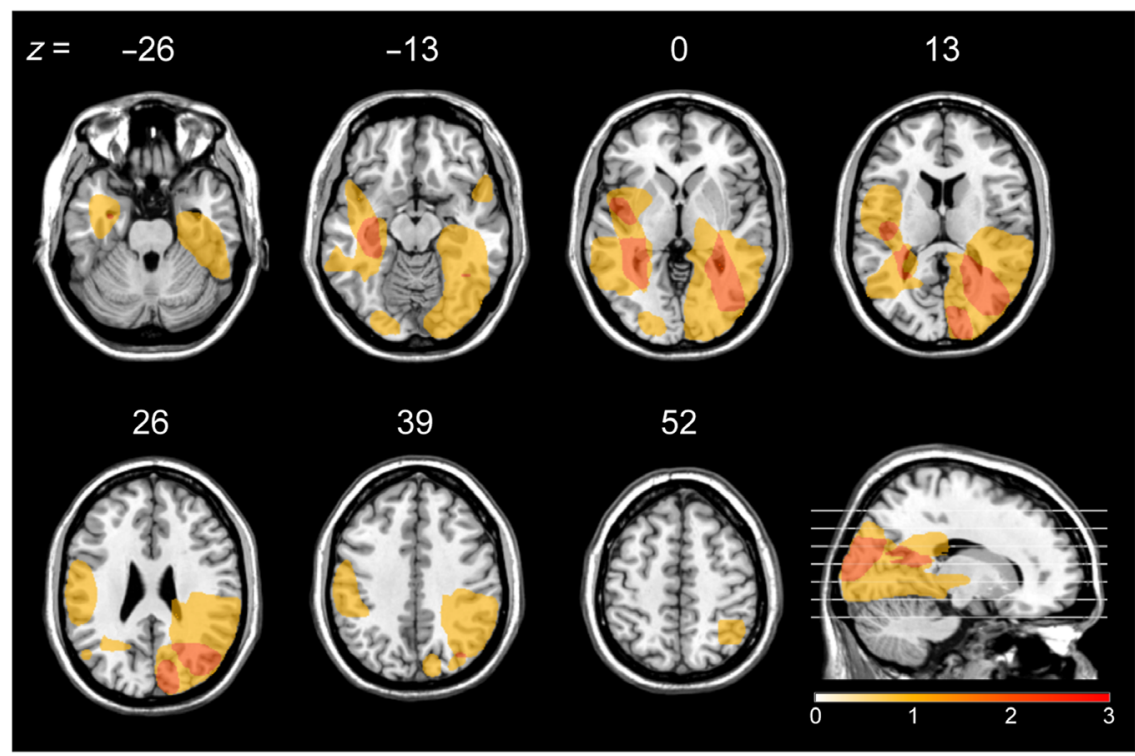

Table 4. Results of the Neuropsychological Tests for the Two Groups of Participants

\begin{tabular}{|c|c|c|c|c|}
\hline Group & $T M T B-A(\mathrm{sec})$ & MCST Cat. & MCST PE & FAB Score \\
\hline vmPFC patients & $96(28) * *$ & $4(2)^{* *}$ & $9(8) * *$ & $14(3) * * *$ \\
\hline Other PFC patients & $87(40)^{* *}$ & $3(2) * *$ & $10(8)^{* *}$ & $16(3)$ \\
\hline Control lesion patients & $103(49) * *$ & $4(2)^{*}$ & $7(7)$ & $16(2)$ \\
\hline Healthy controls & $37.96(24.53)$ & $6.04(0.56)$ & $1.78(1.97)$ & $17.35(0.83)$ \\
\hline
\end{tabular}

The line labeled TMT B-A reports the average completion time for the Part B minus Part A of the Trail-Making Task. MCST Cat. is the average number of categories completed in the Modified Card Sorting Task, MCST PE is the average number of perseverative errors in the Modified Card Sorting Task, and FAB Score is the Frontal Assessment Battery average score. Numbers in parentheses are the standard deviations. * $p<$ $.05, * * p<.01, * * p<.001$ based on a Dunn's test comparing each patient group and the healthy control group, with a Benjamini-Hochberg adjustment for multiple comparisons. 
Figure 2. Experimental task. In the private condition, the participant chose one lottery and then saw an arrow spinning in both lotteries. The participant saw the outcome of both lotteries (complete condition). In the example presented here, they won $€ 5$, but they could have won $€ 20$ had she chosen the other lottery. In the social condition, a yellow dotted line representing the other participant was displayed in addition to the blue dotted line, so that the participant knew she would see the other's choice. The choice of the other was displayed only after the participant had indicated her own choice. Participants knew that the lotteries were drawn only once; therefore, the same lottery outcomes were displayed on both players' screen.

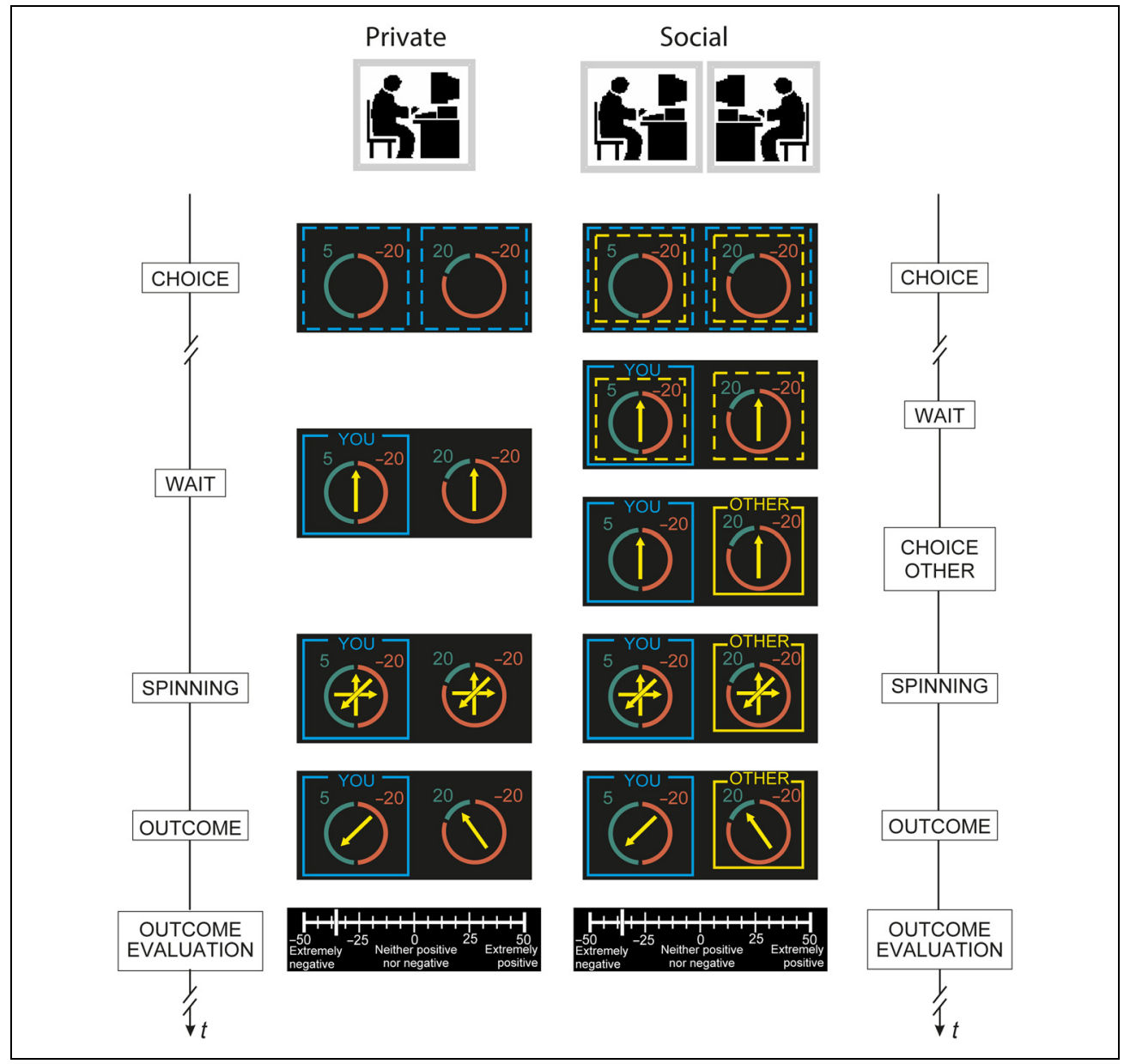

spinning and outcome revelation occurred for the chosen lottery only, whereas neither the outcome of the unchosen lottery nor the choice nor the outcome of the second participant was revealed.

\section{Counterpart's Behavior}

During training, participants saw their counterpart's real choices. During the rest of the session, however, the counterpart's choices were computer simulated. This procedure allowed us, first, to analyze the participant's behavior independently from the counterpart's and, second, to control for the environment created by the other player's choice behavior and outcomes. The computer simulated a risk-averse decision maker, as this choice pattern has been proved to elicit competitive behavior, resulting in participants choosing differently than the computer more often than if it was maximizing expected values (Bault et al., 2008, 2011). The computer selected the lottery with the lowest standard deviation (as defined in Bault et al., 2011, equation S4) in 90\% of the trials. During debriefing, at the end of the experiment, no participant reported any doubt about seeing the actual choice and outcome of the other participant.

\section{Payment}

To discourage participants to mentally sum their earnings and to enable them to treat trials independently, they were told that the outcome from 20 randomly drawn trials would be cumulated and that, at the end of the experiment, they would receive this amount plus a $€ 5$ show-up fee. However, to comply with the ethical committee policy, all participants were offered $€ 20$, irrespective of their gains in the game. When the hospital policy did not allow patients to receive monetary compensation, they were offered gift tokens of the same amount.

\section{Statistical Analysis}

\section{Subjective Ratings Analysis}

After the counterpart's choice and the outcome of the lotteries were revealed to the participants, several events were possible. Trials were categorized as follows according to the condition: private partial feedback (PP), private complete feedback (PC), social same choice (SSC; when the participant and the counterpart chose the same lottery), and social different choice (SDC; when they chose different lotteries). Social trials always received complete 
feedback, even when both players selected the same lottery. Trials were also categorized as relative gain $(+)$ or relative loss (-) trials, depending on the sign of the difference between the outcomes of the chosen and unchosen lottery. In partial feedback trials, we considered the difference between the obtained and nonrealized outcomes of the chosen lottery.

Nonparametric tests were applied, because several parametric assumptions (particularly normal distribution of errors) were violated. The significance of the difference between behavioral variables, RT, and subjective ratings was estimated with the Wilcoxon signed-rank test (WSRT) using Stata (StataCorp LLC, College Station, TX). One-tailed tests were used when we had strong hypothesis on the directionality of the effect $\{|\mathrm{PP}|,|\mathrm{SSC}|\}$ $<\mid$ PC $|<|$ SDC $\mid$ (Bault et al., 2008, 2011). Betweengroup differences were tested with Kruskal-Wallis tests. Post hoc planned comparisons between groups were made with a Dunn's test, with a Benjamini-Hochberg adjustment for multiple comparisons.

One vmPFC patient was excluded from the subjective rating analysis because he failed to report them, answering 0 (the default value) in $52.5 \%$ in the trials, within $0.78 \mathrm{sec}$ on average (1.59 sec for all trials as opposed to an average of $7.66 \mathrm{sec}$ for the other patients).

\section{Choice Behavior Analysis}

We analyzed how the expected value of lotteries anticipated disappointment and regret influenced choices in all conditions. The variables difference in expected value $(\mathrm{dEV})$, regret $(r)$, and disappointment $(d)$ are defined following Camille et al. (2004):

$$
\begin{aligned}
\mathrm{dEV} & =\mathrm{EV}_{1}-\mathrm{EV}_{2}=\left[p x_{1}+(1-p) y_{1}\right]-\left[q x_{2}+(1-q) y_{2}\right] \\
d & =\left[\left|y_{2}-x_{2}\right|(1-q)\right]-\left[\left|y_{1}-x_{1}\right|(1-p)\right] \\
r & =\left|y_{2}-x_{1}\right|-\left|y_{1}-x_{2}\right|
\end{aligned}
$$

where $x_{1}, y_{1}$ and $x_{2}, y_{2}$ are the two possible outcomes of the first and the second lotteries, respectively, with $x_{1}>$ $y_{1}$ and $x_{2}>y_{2}$. The probability of $x_{1}$ is $p$, and the probability of $y_{1}$ is $(1-p)$. The probability of $x_{2}$ is $q$, and the probability of $y_{2}$ is $(1-q)$.

We estimated the probability of the participant choosing lottery 1 as a function of the difference in expected value $(\mathrm{dEV})$, anticipated regret $(r)$, and disappointment $(d)$.

$$
\operatorname{Pr}(c=1 \mid c)=\frac{1}{1+e^{\propto+\beta . \operatorname{dEV}+\gamma \cdot r+\delta . d}}
$$

A positive (negative) and significant expected value (dEV) coefficient indicates that subjects were more likely to choose the lottery with highest (lowest) expected value. Anticipated disappointment $(d)$ is related to the perspective of getting the lowest outcome of the chosen lottery in comparison with the highest outcome of the same lottery. This distance in absolute value is weighted by the probability of the lowest outcome, assuming that participants attempt to avoid highly probable losses. Anticipated disappointment depends on the range of the lotteries' possible outcomes, which is similar to a measure of risk propensity. Anticipated regret is based on the consideration of a possible choice alongside the rejection of its alternatives. The process of minimizing the anticipated regret (denoted as $r$ ) consists of rejecting the lottery associated with highest regret propensity, when comparing the lowest outcome of this lottery and the highest outcome of the alternative lottery. In social trials, anticipated regret can be considered as anticipated envy, as previous research has shown that participants' behavior is driven by social comparison (Bault et al., 2008). Positive coefficients for $r$ and $d$ indicate that participants consistently anticipated (minimized) regret and disappointment, respectively.

Choice behavior was analyzed with multilevel mixed logit regressions with participants nested in groups, which allows us to estimate both random and conditional fixed effects. Parameters were estimated by maximum likelihood. All regressions were run using the statistical software package Stata (StataCorp).

For the VLSM analyses, the same regressions (modeling choice as a function of $\mathrm{dEV}, d$, and $r$ ) were run at the individual level in private complete and social trial separately. The $r$ coefficients were then used as behavioral predictor in the VLSM analysis. For one patient (Patient 11 in Table 2), the estimation failed because of multicollinearity. The model was reestimated excluding anticipated disappointment $(d)$ from the regression for this patient. The $r$ coefficient was used for VLSM analyses (see below) as for the other participants.

\section{Voxel-based Lesion-Symptom Mapping}

VLSM analyses (Bates et al., 2003) were performed using the Brunner-Munzel rank-order test implemented in Niistat (https://www.nitrc.org/projects/niistat/). This analysis compares coefficients of patients having versus not having a lesion in a given voxel. The eight patients with lesions of PFC nonrestricted to the vmPFC were included in this analysis, in addition to the vmPFC and lesion control patients. Only voxels that were lesioned in at least three patients were considered.

Statistics were computed using the general linear model. The threshold of resulting statistical maps were subsequently set at $p<.05$ using permutation-derived correction ( $Z=2.82$ for the subjective ratings analysis). Power analysis revealed sufficient power in the vmPFC, dorsomedial PFC (dmPFC), and left lateral OFC. Power was not sufficient in the right lateral OFC.

In our first analysis, we used the difference in subjective ratings between the $\mathrm{PP}^{-}$and $\mathrm{PC}^{-}$events as behavioral measure. This measure controls for interindividual variability in the use of the rating scale. For the choice analysis, we used the coefficients for anticipated regret 
extracted from individual logistic regressions with all complete feedback trials.

\section{RESULTS}

\section{Subjective Ratings}

\section{Effect of Counterfactual Comparison: Partial Feedback versus Complete Feedback in Private Trials}

We first replicated the results of Camille et al. (2004) concerning the role of the vmPFC in regret (or private counterfactual) processing. In the partial feedback condition, disappointment ( $\mathrm{PP}^{-}$events) is characterized by the unfavorable comparison between the obtained outcome and the unobtained outcome from the chosen lottery. In complete feedback condition, regret ( $\mathrm{PC}^{-}$events) is operationalized as the unfavorable comparison between the outcome of the chosen lottery and the foregone outcome of the unchosen lottery (upward counterfactual). For a given obtained outcome, we expected a stronger effect of regret compared with disappointment (i.e., amplification effect).

In the healthy control group, emotional reactions to relative losses were stronger in the complete feedback condition than in the partial feedback condition (WSRT $\mathrm{PC}^{-}<\mathrm{PP}^{-}, Z=1.93, p=.03$; Figure 3A). The lesion

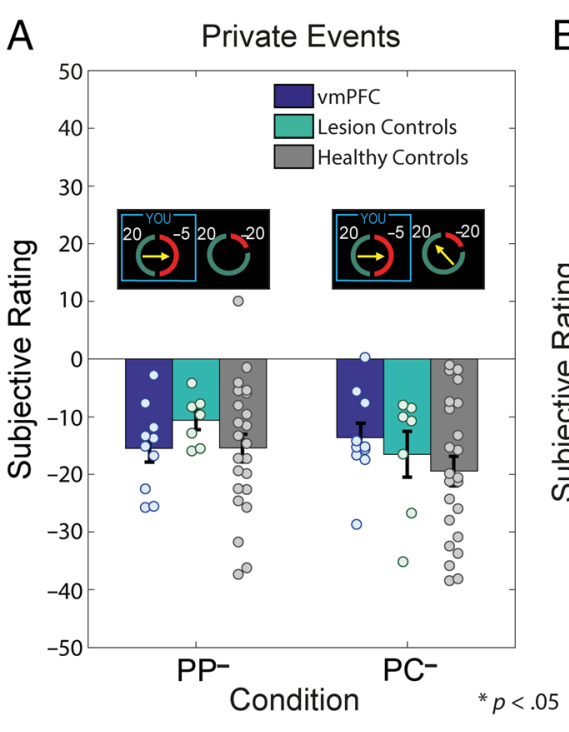

C

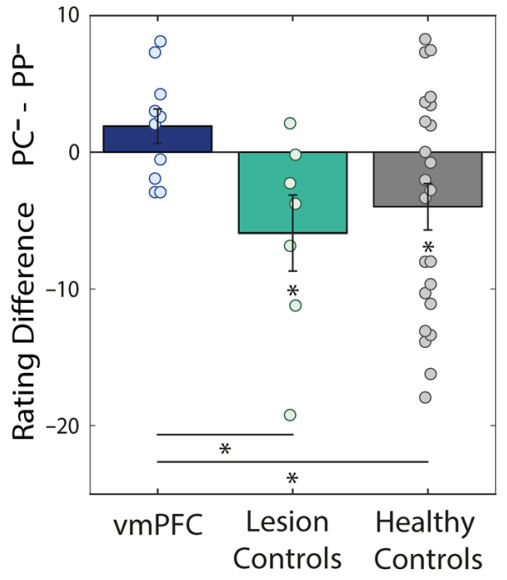

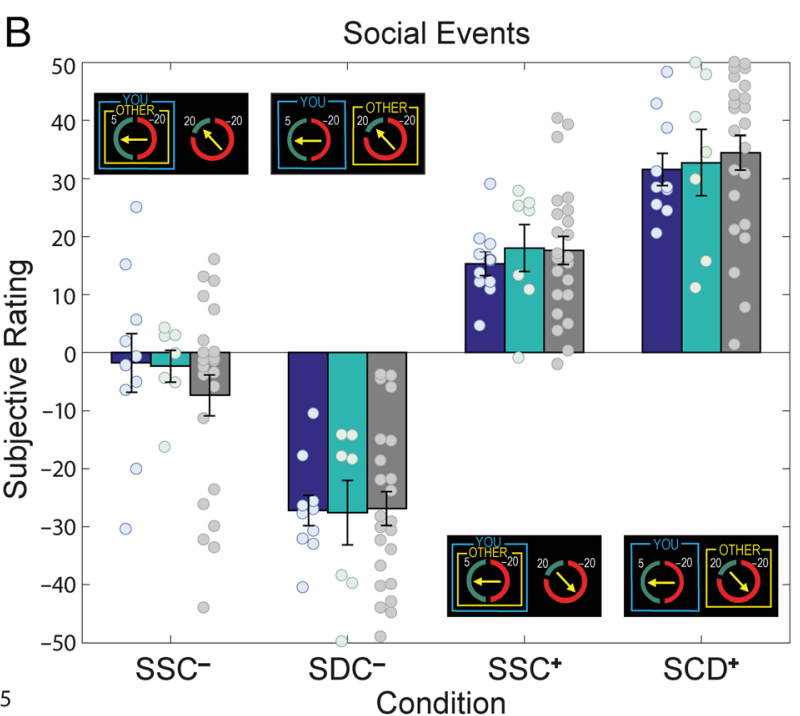

$\mathrm{D}$

Social Comparison Effect

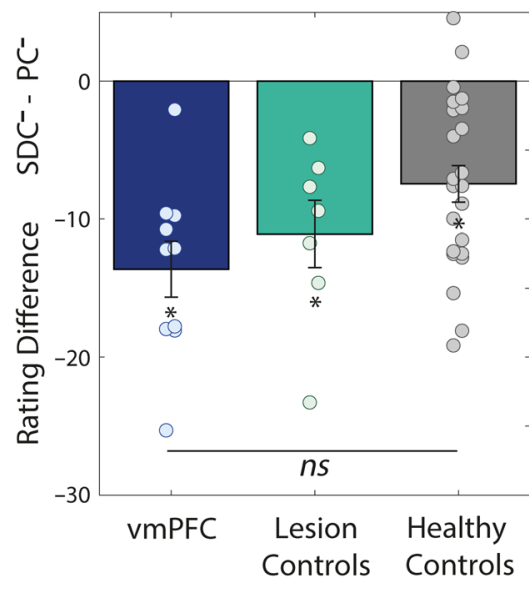

Figure 3. Subjective ratings in private events and social events. (A) Mean subjective ratings given by the vmPFC patients, lesion control patients, and healthy control participants in the partial and complete feedback conditions for negative events. The control group gave lower subjective ratings in PC trials than in partial feedback trials, whereas the vmPFC patients did not. $\mathrm{PP}=$ private condition/partial feedback; $\mathrm{PC}=$ private condition/complete feedback. - signs stand for relative losses. (B) Mean subjective ratings for the vmPFC patients, lesion control patients, and healthy control participants in the social condition (complete feedback). Negative (positive) events include all trials in which the obtained outcome was lower (higher) than the foregone outcome. The vmPFC patient group did not differ from any of the control groups for all four events. SSC = social condition/same choice made by both players; SDC = social condition/different choice. + and - signs stand for relative gain and losses, respectively. (C) Mean subjective ratings distance between the negative PC and PP trials for the three groups. (D) Mean subjective ratings distance between the $\mathrm{SDC}^{-}$and $\mathrm{PC}^{-}$events for the three groups. Circles represent individual data points. Error bars represent SEM. 
control group showed the same effect (WSRT $\mathrm{PC}^{-}<\mathrm{PP}^{-}$, $Z=1.94, p=.03)$, whereas the vmPFC patients did not (WSRT PC ${ }^{-}<\mathrm{PP}^{-}, Z=-1.42, p=.92$ ). However, there was no significant effect of group for subjective ratings of $\mathrm{PC}^{-}$events (Kruskal-Wallis, $\chi^{2}=1.775, p=.4118$ ).

To control for the frequencies of specific pairs of obtained/unobtained outcomes that could differ between groups (due to behavioral differences), we restricted the analysis to trials in which, for a given obtained outcome of -5 or +5 , subjective ratings could potentially be modulated by higher $(+20)$ or lower $(-20)$ unobtained outcomes (Figure 4). The subjective ratings reported by the vmPFC patients were not modulated at all by the feedback on the outcome of the unchosen gamble. vmPFC patients still evaluated a gain (loss) of $€ 5$ positively (negatively) even when they could have won (loss) more choosing the alternative option. The three groups differed in the subjective rating of $\mathrm{PC}^{-}$events in the restricted data set (Kruskal-Wallis, $\chi^{2}=7.672, p=.0216$ ). Pairwise comparisons revealed that healthy control participants reported stronger negative ratings when obtaining 5 or -5 in the view of a missed opportunity to get $€ 20$ than vmPFC patients (Dunn's test, $Z=2.603, p=.014$ ). The lesion control and healthy control groups did not differ significantly (Dunn's test, $Z=1.522, p=.096$ ).
Note that, similar to the results previously reported (Camille et al., 2004), the impairment in vmPFC patients was restricted to the negative domain. The subjective ratings for $\mathrm{PC}^{+}$events did not differ in the three groups (Kruskal-Wallis, $\chi^{2}=4.309, p=.116$ ). These effects were confirmed by a mixed linear regression that used the four lottery values as predictors of subjective ratings (Table 5). Although subjective ratings of healthy controls were decreased by the outcome of the unchosen lottery in PC trials, the ratings of vmPFC patients were unaffected and the group interaction was significant. The lesion control group also differed from healthy controls for both the obtained outcome and outcome of the unchosen lottery, suggesting that the difference was not specific to regret. Indeed, the group interaction disappeared when we restricted the analysis to regret events (Outcome chosen < Outcome unchosen, Outcome unchosen $\times$ Group, $\beta=-0.318, p=.096)$, whereas it remained significant for vmPFC patients (Outcome unchosen $\times$ Group, $\beta=-0.431, p=.007)$.

Finally, we controlled for a potential effect of medication on regret impairment. Patients under psychoactive medication did not differ from medication-free patients in their ratings in $\mathrm{PP}^{-}$and $\mathrm{PC}^{-}$events (Mann-Whitney, $\left.\mathrm{PP}^{-}: Z=0.30, p=.76 ; \mathrm{PC}^{-}: Z=0.91, p=.36\right)$.
Figure 4. Effect of unobtained outcomes on mean subjective ratings given by the $\mathrm{vmPFC}$ patients and healthy control participants in the partial and complete feedback conditions. Mean subjective ratings reported by control participants and $v m P F C$ patients for two obtained outcomes ( -5 or 5 ) as a function of the unobtained outcome (blue line, -20 ; green line, +20$)$ in the two private conditions. In the partial condition, the unobtained outcome corresponds to the unobtained value of the chosen gamble. In the complete condition, it corresponds to the outcome of the nonchosen gamble. Error bars represent SEM.

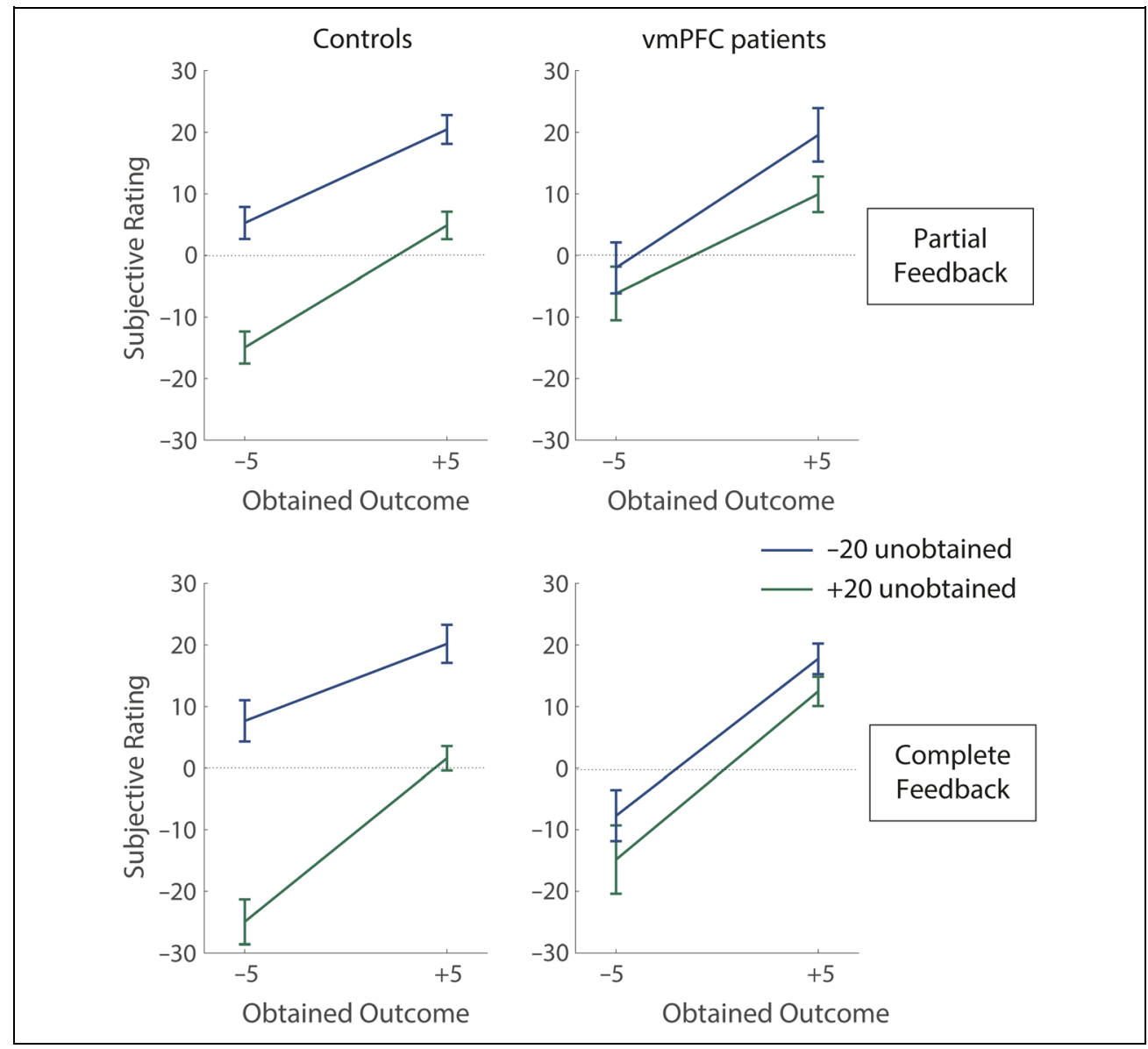


Table 5. Effect of Counterfactual Comparison on Subjective Ratings in the Private Condition

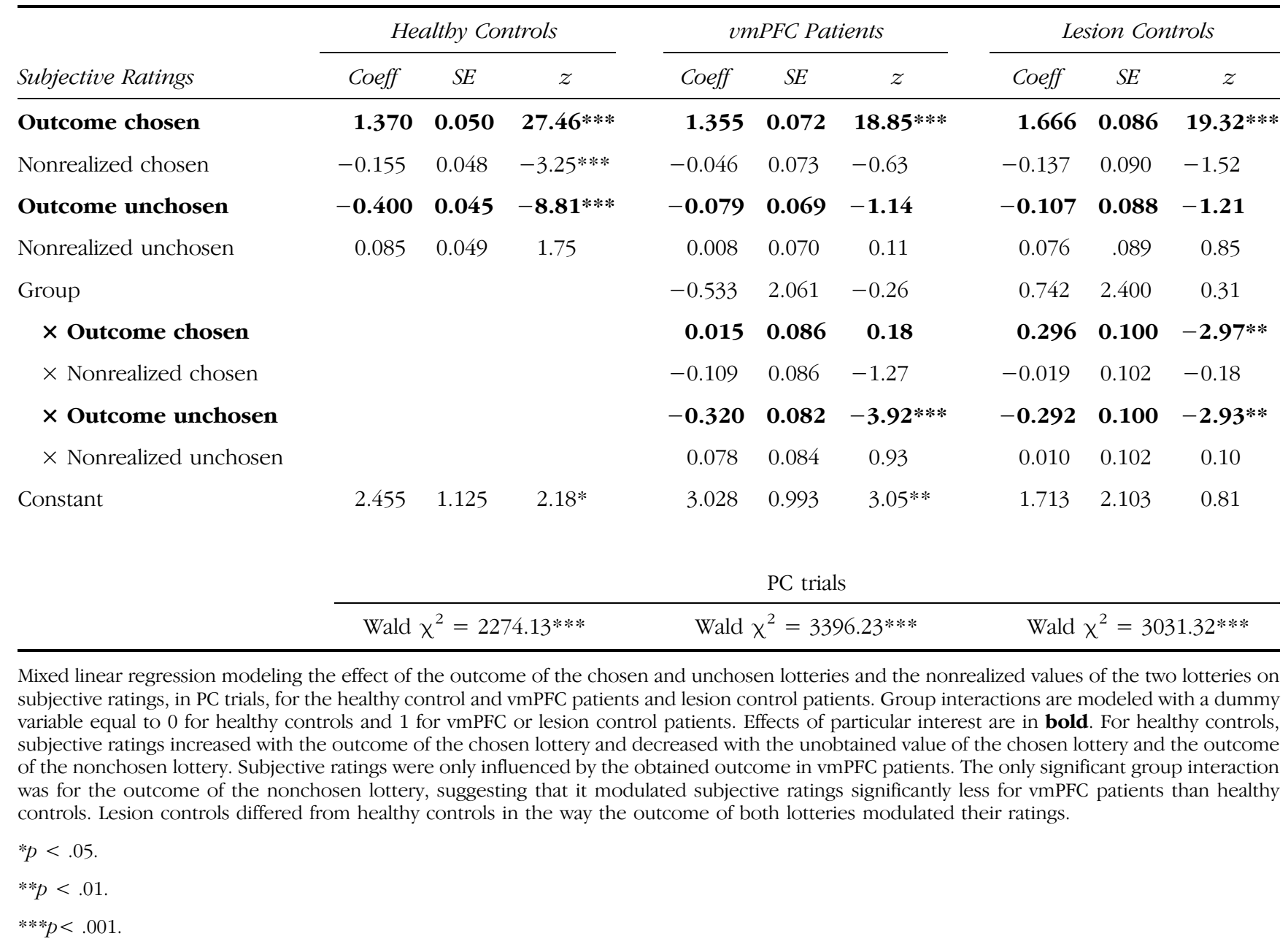

Therefore, we exclude that psychoactive drugs can account for the observed impairment in vmPFC patients.

In summary, unlike healthy controls and lesion control patients, vmPFC patients did not take into account the outcome of the unchosen option when evaluating the outcome of their choice.

\section{Involvement of Different Regions of the PFC in Regret Impairment}

We ran a VLSM analysis to better characterize which region of the PFC is critical to report stronger subjective feelings caused by counterfactual thinking after a bad decision. The analysis was run on all patients, including the eight PFC patients with lesions that were not restricted to the vmPFC.

We used the regret amplification variable (difference in subjective ratings between the $\mathrm{PP}^{-}$and $\mathrm{PC}^{-}$events) as behavioral measure in the analysis. No voxel was associated with reduced regret after correction for multiple comparisons. Uncorrected analysis revealed that damage in the medial OFC was associated with impair- ment in reporting regret (MNI coordinates $-3,53,-5$; $Z=2.54)$.

\section{Effect of Social Comparison: Private versus Social Trials}

Social comparison modulated how participants rated the outcome of their choices (Figure 3B). Healthy control participants reported more negative ratings when they lost in the presence of the other player's gain than when they lost in isolation $\left(\mathrm{SDC}^{-}<\mathrm{PC}^{-}\right.$: WSRT $Z=3.76, p<$ .001) or when they lost the same amount as the other participant $\left(\mathrm{SDC}^{-}<\mathrm{SSC}^{-}\right.$, WSRT $Z=3.90, p<.001$ ). They also reported more positive ratings when they won more than their counterpart, compared with winning in isolation $\left(\mathrm{SDC}^{+}>\mathrm{PC}^{+}\right.$: WSRT $Z=4.15, p<.001$ ) or winning the same amount $\left(\mathrm{SDC}^{+}>\mathrm{SSC}^{+}\right.$: WSRT $Z=$ $4.18, p<.001)$. Thus, healthy control participants were sensitive to social comparison signals, deriving strong pleasure from outperforming their counterparts and strong displeasure from being outperformed.

Patients with vmPFC lesion displayed the exact same pattern of responses in the social settings. As for the healthy 
control participants, $\mathrm{SDC}^{-}$events were rated more negatively than $\mathrm{PC}^{-}$events (WSRT, $Z=2.75, p=.003$ ) or SSC $^{-}$events (WSRT, $Z=2.75, p=.003$ ). SDC $^{+}$events elicited more positive emotions than $\mathrm{PC}^{+}$(WSRT, $Z=$ $2.75, p=.003$ ) and SSC ${ }^{+}$(WSRT, $Z=2.65 p=.004$ ).

The lesion control patient group was sensitive to negative social comparison signals as well (WSRT, SDC $^{-}$vs. $\mathrm{PC}^{-}: Z=2.28, p=.01 ; \mathrm{SDC}^{-}$vs. $\mathrm{SSC}^{-}: Z=2.28, p=$ $.01)$ and to some extent to positive social comparison signals (WSRT, $\mathrm{SDC}^{+}$vs. $\mathrm{PC}^{+}: Z=1.44, p=.07$; $\mathrm{SDC}^{+}$ vs. $\mathrm{SSC}^{+}: Z=1.94, p=.03$ ).

The three groups gave similar ratings for all social events (Kruskal-Wallis, SSC ${ }^{-}: \chi^{2}=.502, p=.778$; SDC $^{-}: \chi^{2}=$ $0.038, p=.981 ; \mathrm{SSC}^{+}: \chi^{2}=0.556, p=.757$; and $\mathrm{SDC}^{+}:$ $\left.\chi^{2}=0.977, p=.614\right)$. In summary, we did not find any difference in the counterfactual evaluation of the outcomes in social settings between vmPFC patients, lesion control patients, and healthy control participants.
These effects were confirmed by a mixed linear regression that used the four lottery values as predictors of subjective ratings in social trials (Table 6). Subjective ratings were influenced by the outcomes of both the chosen and unchosen lotteries in all three groups. The influence of the outcome of the unchosen lottery was not significantly different between the two lesion groups and the healthy control group. Finally, the effect of the outcome of the unchosen lottery on subjective ratings was significantly higher in the social than in the private condition (interaction: Private $\times$ Outcome unchosen) for the vmPFC patient group.

\section{Emotional Amplification Effects in Private and Social Trials}

The signature of regret is the emotional amplification observed in $\mathrm{PC}^{-}$trials compared with $\mathrm{PP}^{-}$trials (Figure 3C).

Table 6. Effect of Counterfactual Comparison on Subjective Ratings in the Social Condition

\begin{tabular}{|c|c|c|c|c|c|c|c|c|c|}
\hline \multirow[b]{2}{*}{ Subjective Ratings } & \multicolumn{3}{|c|}{ Healthy Controls } & \multicolumn{3}{|c|}{ vmPFC Patients } & \multicolumn{3}{|c|}{ Lesion Controls } \\
\hline & Coeff & $S E$ & $z$ & Coeff & $S E$ & $z$ & Coeff & $S E$ & $z$ \\
\hline Outcome chosen & 1.427 & 0.091 & $15.69 * * *$ & 1.374 & 0.060 & $22.99 * * *$ & 1.586 & 0.073 & $21.68 * * *$ \\
\hline Nonrealized chosen & 0.115 & 0.121 & 0.95 & -0.097 & 0.07 & -1.44 & -0.0378 & 0.085 & -0.45 \\
\hline Outcome unchosen & -0.694 & 0.109 & $-6.36 * * *$ & -0.322 & 0.105 & $-3.08 * *$ & -0.272 & 0.135 & $-2.01 *$ \\
\hline Nonrealized unchosen & 0.250 & 0.075 & $3.34 * * *$ & 0.037 & 0.058 & 0.65 & 0.076 & .089 & 0.85 \\
\hline Private & & & & 4.217 & 1.338 & $3.15 * *$ & 0.707 & 1.753 & 0.40 \\
\hline$\times$ Outcome unchosen & & & & 0.290 & 0.110 & $2.63 * *$ & 0.101 & 0.141 & 0.71 \\
\hline Group & & & & 3.174 & 2.290 & 1.39 & 1.171 & 2.796 & 0.42 \\
\hline$\times$ Outcome chosen & & & & -0.011 & 0.072 & -0.16 & -0.224 & 0.085 & $-2.62 * *$ \\
\hline$\times$ Nonrealized chosen & & & & -0.036 & 0.079 & -0.45 & -0.095 & 0.096 & -0.99 \\
\hline$\times$ Outcome unchosen & & & & -0.183 & 0.126 & -1.45 & -0.233 & 0.156 & -1.50 \\
\hline$\times$ Nonrealized unchosen & & & & 0.097 & 0.069 & 1.39 & 0.010 & 0.102 & 0.10 \\
\hline$\times$ Private & & & & -3.807 & 1.603 & $-2.37 *$ & -0.298 & 2.00 & -0.15 \\
\hline$\times$ Outcome unchosen $\times$ Private & & & & -0.184 & 0.133 & -1.38 & 0.006 & 0.163 & 0.04 \\
\hline Constant & 1.920 & 1.217 & 1.58 & -1.200 & 1.908 & -0.63 & 0.805 & 2.449 & 0.33 \\
\hline & \multicolumn{3}{|c|}{ SDC trials } & \multicolumn{3}{|c|}{ PC and SDC trials } & \multicolumn{3}{|c|}{ PC and SDC trials } \\
\hline & \multicolumn{3}{|c|}{ Wald $\chi^{2}=1491.50 * * *$} & \multicolumn{3}{|c|}{ Wald $\chi^{2}=5835.26 * * *$} & \multicolumn{3}{|c|}{ Wald $x^{2}=4741.12 * * *$} \\
\hline
\end{tabular}

Mixed linear regression modeling the effect of social comparison on subjective ratings in healthy controls, vmPFC patients, and lesion controls. Interactions with group in SDC trials are modeled with a dummy variable equal to 0 for healthy controls and 1 for patients. Interactions with conditions are modeled with a dummy variable equal to 1 for private trials and 0 for SDC trials. Effects of particular interest are in bold. For all groups, subjective ratings increased with the outcome of the chosen lottery and decreased with the outcome of the unchosen lottery. Subjective ratings were influenced by the outcome of the unchosen lottery significantly more in SDC than in PC trials in vmPFC patients (significant interaction: Private $\times$ Outcome unchosen).

$* p<.05$.

$* * p<.01$.

$* * * p<.001$. 
Similarly, the signature of envy is the emotional amplification observed in $\mathrm{SDC}^{-}$trials compared with $\mathrm{PC}^{-}$trials (Figure 3D).

The difference in subjective ratings between $\mathrm{PC}^{-}$and $\mathrm{PP}^{-}$(Kruskal-Wallis $\chi^{2}=5.391, p=.067$ ) was smaller for vmPFC patients than for the healthy controls (all trials, Dunn's test, $Z=1.90, p=.043$ ) and the lesion control patients (Dunn's test, $Z=2.14, p=.048$ ), whereas the healthy and lesion control groups did not differ (Dunn's test, $Z=0.78, p=.21$ ). The amplification effect between $\mathrm{PC}^{-}$and $\mathrm{SDC}^{-}$events was comparable for the three groups (Kruskal-Wallis, $\chi^{2}=4.949, p=.084$ ), so was the amplification effect between $\mathrm{PC}^{+}$and $\mathrm{SDC}^{+}$ events $\left(\chi^{2}=0.323, p=.851\right)$.

vmPFC patients experienced a higher emotional amplification elicited by social comparison relative to private counterfactual comparison $\left(\mathrm{PC}^{-}-\mathrm{SDC}^{-}>\mathrm{PP}^{-}-\mathrm{PC}^{-}\right.$, WSRT, $Z=2.70, p=.007)$. This was not the case for the healthy (WSRT, $Z=1.49, p=.136$ ) and lesion (WSRT, $Z=1.69, p=.091$ ) control groups. The difference in amplification effect was significantly affected by the group variable (Kruskal-Wallis, $\chi^{2}=7.781, p=$ .020). The vmPFC group differed from both the healthy (Dunn's test, $Z=2.74, p=.009$ ) and lesion controls (Dunn's test, $Z=1.90, p=.043$ ). The two control groups did not significantly differ (Dunn's test, $Z=0.23$, $p=$ .408).

\section{Choice Behavior}

We tested a model of choice incorporating the difference in expected values between the two gambles, anticipated disappointment and regret, as choice predictors in private and social trials. Anticipated regrets in private and social trials are both defined on the same counterfactual comparison between expected outcomes of chosen and unchosen options; the only difference between the two situations is the players' knowledge that the outcome of the alternative lottery could be obtained by the other participant.

Mixed logistic regressions showed that, in addition to maximizing expected values, healthy control participants significantly maximized expected values $\left(\beta_{\mathrm{EV}}=0.131\right.$, $p<.001)$ and minimized future regret $\left(\beta_{\mathrm{r}}=0.042, p<\right.$ .001 ) in complete feedback trials (Table 7 ).

vmPFC patients chose the lottery with highest expected value $\left(\beta_{\mathrm{EV}}=0.070, p=.004\right)$. Although they maximized expected values less than healthy controls $\left(\beta_{\mathrm{EV} \times \text { Group }}=0.061, p=.033\right)$; it was the strongest predictor of their choice. This suggests that they understood the game and were as motivated to earn money. They took into account potential future regret as well $\left(\beta_{\mathrm{r}}=\right.$ $0.015, p=.02)$. However, they did so significantly less than healthy controls $\left(\beta_{\mathrm{r} \times \text { Group }}=0.027, p=.001\right)$. We tested the hypothesis that $\mathrm{vmPFC}$ anticipated regret

Table 7. Choice Behavior in the Complete Feedback Condition

\begin{tabular}{|c|c|c|c|c|c|c|c|c|c|}
\hline \multirow[b]{2}{*}{ Choice } & \multicolumn{3}{|c|}{ Healthy Controls } & \multicolumn{3}{|c|}{ vmPFC Patients } & \multicolumn{3}{|c|}{ Lesion Controls } \\
\hline & Coeff & $S E$ & $z$ & Coeff & $S E$ & $z$ & Coeff & $S E$ & $z$ \\
\hline $\mathrm{dEV}$ & 0.131 & 0.015 & $8.44 * * *$ & 0.067 & 0.024 & $2.89 * *$ & 0.009 & 0.028 & 0.33 \\
\hline$d$ & 0.006 & 0.007 & 0.96 & -0.037 & 0.010 & $-3.55 * * *$ & 0.049 & 0.032 & 0.42 \\
\hline $\boldsymbol{r}$ & 0.042 & 0.004 & $9.30 * * *$ & 0.015 & 0.006 & $2.38 *$ & 0.039 & 0.014 & $4.89 * * *$ \\
\hline Group & & & & -0.032 & 0.089 & 0.36 & 0.251 & 0.123 & $2.04 *$ \\
\hline$\times \mathrm{dEV}$ & & & & 0.061 & 0.029 & $2.13 *$ & 0.123 & 0.032 & $3.86^{*}$ \\
\hline$\times d$ & & & & 0.044 & 0.012 & $3.52 * * *$ & 0.001 & 0.014 & 0.11 \\
\hline$\times \boldsymbol{r}$ & & & & 0.027 & 0.008 & $3.44 * * *$ & 0.003 & 0.009 & 0.39 \\
\hline \multirow[t]{3}{*}{$\times$ Constant } & 0.041 & 0.049 & 0.83 & 0.077 & 0.107 & 0.72 & -0.210 & 0.107 & -1.95 \\
\hline & \multicolumn{3}{|c|}{$\begin{array}{l}\mathrm{LL}=-1180.89 \\
\text { Wald } \chi^{2}=165.71 * * *\end{array}$} & \multicolumn{3}{|c|}{$\begin{array}{l}\mathrm{LL}=-1684.54 \\
\text { Wald } \chi^{2}=206.39 * * *\end{array}$} & \multicolumn{3}{|c|}{$\begin{array}{l}\mathrm{LL}=-1545.69 \\
\text { Wald } \chi^{2}=205.74 * * *\end{array}$} \\
\hline & \multicolumn{9}{|c|}{ Complete Trials, $N=80$} \\
\hline
\end{tabular}

Mixed logistic regression modeling the effect, on choice behavior, of the difference in expected value (dEV), anticipated disappointment $(d)$, anticipated regret $(r)$ between the lotteries, in complete feedback trials. Effects of particular interest are in bold. The group variable is equal to 1 for controls and 0 for patients. The interactions between $\mathrm{dEV}, d, r$, and group are also included. vmPFC patients anticipated regret significantly less than healthy controls in their decision.

$* p<.05$.

$* * p<.01$.

$* * * p<.001$. 
more in social than private trials; however, it was not the case-the condition interaction was not significant $\left(\beta_{\mathrm{r} \times \text { Condition }}=0.001, p=.917\right)$. vmPFC patients minimized future disappointment (variable $d$ in the model) in their choices in private trials. This could reflect risktaking behavior typical in such patients (the disappointment variable of our model is similar to risk as measured in economics and finance, i.e., variance of the potential outcomes).

Lesion control patients did not maximize expected values $\left(\beta_{\mathrm{EV}}=0.009, p=.743\right)$, showing reduced performance in the choice task, but they did anticipate regret $\left(\beta_{\mathrm{r}}=0.038, p<.001\right)$. When compared with the healthy control group, they relied on expected values to make their choice significantly less than the healthy control group $\left(\beta_{\mathrm{EV} \times \text { Group }}=0.123, p<.001\right)$, but they did not differ in regret $\left(\beta_{\mathrm{r} \times \text { Group }}=0.003, p=.698\right)$.

\section{Choice Adaptation}

We next tested the hypothesis that the social context facilitated regret anticipation in vmPFC patients during the course of the task. We found a significant time trend (random effect logistic regression $\beta_{\mathrm{r} \times \text { Trial }}=0.0003, z=2.14$, $p<.032$ ), where vmPFC patient anticipated regret increasingly during the course of the task (Figure 5A). When dividing complete feedback trials into three equal parts, we found that regret anticipation was not significant in vmPFC patients during early and middle trials
(Trials 1-26, $\beta_{\mathrm{r}}=0.008, p=.444$; Trials 27-53, $\beta_{\mathrm{r}}=$ $0.016, p=.119$ ) and significantly lower than in the two other groups (Trials $1-26, \beta_{\mathrm{r} \times \text { Group }}=-0.038, p=.003$; Trials 27-53, $\left.\beta_{\mathrm{r} \times \text { Group }}=-0.024, p=.046\right)$. During late trials, however, vmPFC patients significantly anticipated regret (Trials $54-80, \beta_{\mathrm{r}}=0.028, p=.037$ ) and the group interaction was no longer significant (Trials 54-80, $\left.\beta_{\mathrm{r} \times \text { Group }}=-0.010, p=.524\right)$.

We next tested whether this increase of regret anticipation was driven by social trials. We ran a regression looking at the effect of the condition of the preceding trial on regret anticipation. There was a trend for vmPFC patients to anticipate regret more, in their choices, when they have experienced a social trial rather than a private trial before (Figure 5B and Table 8). vmPFC patients significantly minimized future regret in their decisions following a social trial, but not after a private trial.

\section{VLSM Analyses of Choice}

We conducted two VLSM analyses on choice data. We used the coefficients of anticipated regret ( $r$ coefficients in all complete feedback trials) as the behavioral measures in the first and second VLSM analyses. To test the hypothesis that OFC and dmPFC lesions might result in different effects on regret anticipation in social and private trials, we also tested them separately.

No voxel survived correction for multiple comparisons for either analysis. Uncorrected analyses revealed that
Figure 5. Regret anticipation during choices. (A) Evolution of regret anticipation during the course of the task for the three groups. Logistic regressions were run separately for early Trials 1-26, middle Trials 27-53, and late Trials 54-80. vmPFC patients increasingly anticipated regret over time. In early and middle trials, their regret coefficients were not significantly different from zero and were significantly lower than the other two groups. By the end of the task, they significantly anticipated regret in their choices in a way that was statistically undistinguishable from the other two groups. (B) Regret anticipation for vmPFC patients following private and social trials. vmPFC patients minimized future regret in their choices after having experienced a social trial but not after a private trial. Full regressions are shown in Table 8.

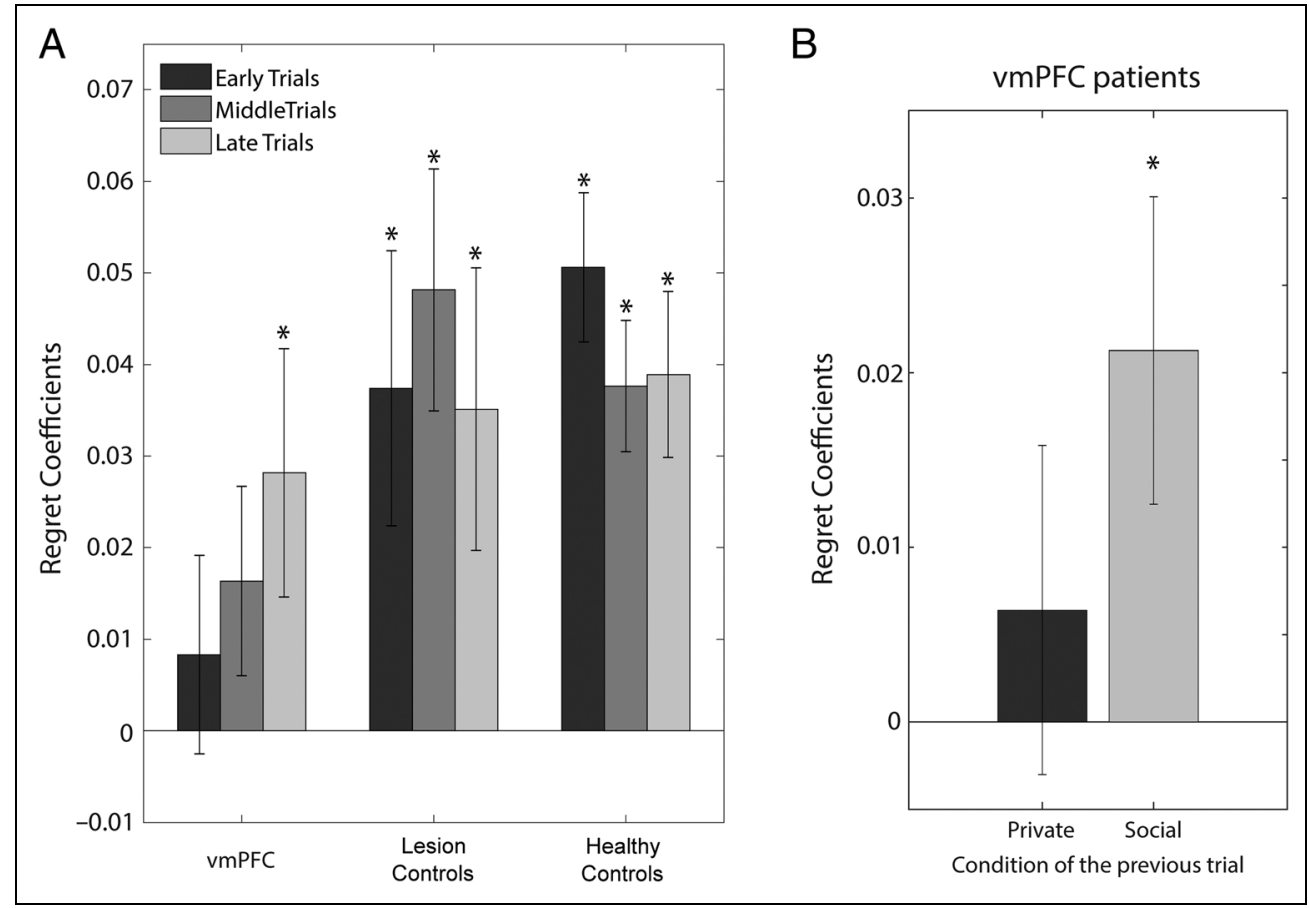


Table 8. Effect of the Condition of the Previous Trial on vmPFC Patients' Choices

\begin{tabular}{|c|c|c|c|c|c|c|c|c|c|c|c|c|}
\hline \multirow[b]{2}{*}{ Choice } & \multicolumn{4}{|c|}{ All Complete Trials } & \multicolumn{4}{|c|}{ Previous Trial $=$ Private } & \multicolumn{4}{|c|}{ Previous Trial $=$ Social } \\
\hline & Coeff & $S E$ & $z$ & $p$ & Coeff & $S E$ & $z$ & $p$ & Coeff & $S E$ & $z$ & $p$ \\
\hline $\mathrm{dEV}$ & 0.076 & 0.025 & 3.08 & .002 & 0.044 & 0.033 & 1.33 & .184 & 0.114 & 0.039 & 2.92 & .003 \\
\hline$d$ & -0.042 & 0.011 & -3.92 & $<.001$ & -0.032 & 0.014 & -2.33 & .02 & -0.059 & 0.018 & -3.23 & .001 \\
\hline $\boldsymbol{r}$ & 0.004 & 0.008 & 0.42 & .671 & 0.006 & 0.009 & 0.68 & .497 & 0.021 & 0.009 & 2.41 & .016 \\
\hline$r \times$ Previous social & 0.019 & 0.010 & 1.9 & .057 & & & & & & & & \\
\hline \multirow[t]{2}{*}{ Constant } & 0.021 & 0.075 & 0.28 & .781 & 0.102 & 0.106 & 0.96 & .335 & -0.043 & 0.110 & -0.39 & .695 \\
\hline & \multicolumn{4}{|c|}{ Wald $\chi^{2}=42.35^{* * *}$} & \multicolumn{4}{|c|}{ Wald $x^{2}=14.91 * *$} & \multicolumn{4}{|c|}{ Wald $\chi^{2}=26.73 * * *$} \\
\hline \multicolumn{13}{|c|}{$\begin{array}{l}\text { Mixed logistic regression modeling the effect of the difference in expected value (dEV), anticipated disappointment }(d) \text {, anticipated regret }(r) \text {, and its } \\
\text { interaction with the condition, private or social, of the preceding trial. Effects of particular interest are in bold. All complete feedback trials were } \\
\text { included in the left regression, the middle and right regressions report the effect of } \mathrm{dEV}, d \text {, and } r \text { separately for trials that were preceded by a private } \\
\text { or social trial. There was a trend for vmPFC patient to take into account anticipated regret in their choice more after having just experienced a social } \\
\text { trial rather than a private trial. Anticipated regret was not significant when the previous trial was private, but it was significant for decisions following a } \\
\text { social trial, irrespective of the condition of the current trial. }\end{array}$} \\
\hline
\end{tabular}

lesions in the medial orbitalfrontal cortex (MNI coordinate $5,45,-20 ; Z=-2.29$ ) were associated with reduced anticipation of regret ( $r$ coefficients) in all trials. Restricting the analysis to private trials yielded to the same results (MNI coordinate 6, 44, -19; $Z=-2.48$ ). No voxel was associated with a deficit in regret anticipation in social trials.

\section{DISCUSSION}

We studied the involvement of the ventromedial PFC in decisions taken in private versus social settings. We considered two aspects of the decision process: (1) the effect of private versus social counterfactual comparison on the subjective evaluation of a decision outcome and (2) the impact of private and social counterfactual information on future decisions. In private settings, we confirmed the existence of an impairment in patients with lesions in the vmPFC in feeling regret after a suboptimal decision, as they did not show typical emotional reactions to upward counterfactual comparison. In social settings, by contrast, patients with vmPFC lesion provided normal subjective ratings when confronted with the outcome received by another person. More specifically, their reported levels of envy were similar to controls, showing a strong emotional amplification induced by social comparison. This suggests that the vmPFC does not critically mediate the effect of social comparison on outcome evaluation. When considering choice behavior, we observed that vmPFC patients avoided future regret less than control participants. During the course of the task, however, they increased their anticipation of regret. In particular, although they did not display clearly distinct choice behaviors in the private and social conditions, they integrated their experience in private and social trials into future choices differently. Even if they did not attempt to avoid regret in choices following private trials, they did use counterfactual information in their decisions in choices following social trials, suggesting that the increase of regret anticipation over time might be driven by social comparison.

After correcting for multiple comparisons, VLSM analyses did not reveal any significant lesion pattern associated with regret deficit. Uncorrected analyses suggested that failure to reason counterfactually during choices might be associated with lesions in the mOFC.

The findings that vmPFC patients are not sensitive to regret signals in private settings confirm the results of a previous study (Camille et al., 2004) on a different cohort of vmPFC patients. Unlike healthy and lesion controls, in private settings, vmPFC patients did not take into account the outcome of the nonchosen lottery when evaluating their outcome, nor did they anticipate potential negative consequences of their choices. Notably, our vmPFC patients have lesions that spare lateral portions of the PFC, and the results of the VLSM analyses confirmed the implication of the medial OFC in the experience and anticipation of regret. Thus, contrary to Levens et al. (2014), we found impairment in regret processing associated with a damaged medial OFC. However, our sample of patients with specific lateral OFC lesions is too small to exclude the possibility that lateral OFC lesions would result in a similar impairment. The regret impairment is consistent with recent evidence of an 
inability to imagine fictitious and future events following vmPFC damage (Bertossi, Aleo, Braghittoni, \& Ciaramelli, 2016). Indeed, the feeling of regret requires considering an alternative reality in which a greater outcome is obtained by making a different choice.

This study revealed, for the first time, that vmPFC patients are unimpaired when confronting the result of their choices compared with those of others, showing normal emotional responses to social comparison. Similarly to healthy and lesion controls, vmPFC patients reported feeling worse after a loss when informed of a counterpart's gain than after a loss without that information. They also evaluated their outcomes more positively when winning more than their counterparts, compared with when they were winning alone. Thus, vmPFC patients' subjective ratings are consistent with envy and gloating, despite their impairment in recognizing these emotions (Shamay-Tsoory, Tibi-Elhanany, \& AharonPeretz, 2007).

One possible explanation for the vmPFC patients' lack of impairment in the social comparison condition during outcome evaluation concerns the visual salience in the task of the lottery chosen by the other participant, which was surrounded by a bright yellow square. Yet if the impairment in the private condition had been due to a lack of attention to the counterfactual information, vmPFC patients would have been impaired for positive counterfactuals as well. Indeed, they would not have been able to report relief if they had not processed the outcome of the unchosen lottery first as healthy participants do (Bault, Wydoodt, \& Coricelli, 2016). Nonetheless, their deficit is specific to negative counterfactuals, because they did report subjective experiences similar to controls for positive events. In addition, they distinguished between the private condition and situations in which the other player chose the same lottery, suggesting that the information on the other's outcome is meaningful for evaluating their own outcome. Thus, we can conclude that, if the social information is more salient, it must derive from its motivational relevance rather than from perceptual aspects. A previous study with five prefrontal patients reported higher skin conductance responses to emotionally charged pictures during an active task, compared with passive viewing (Damasio, Tranel, \& Damasio, 1990). Although all conditions of our task were identically "active," it is possible that the social condition was more engaging for patients, resulting in normal emotional responses.

Notably, the computational operation of comparing an obtained outcome with an alternative outcome is the same in both the private and the social condition. The only difference in the social condition concerns the knowledge that the alternative outcome belongs to somebody else. The dissociation we observed in vmPFC patients between private and social counterfactual processing suggests that different brain networks are involved in integrating these two types of information in the valuation process.
Our previous fMRI findings (Bault et al., 2011) showed that the mOFC encoded the difference between obtained and foregone outcome the same regardless of the condition (private or social). By contrast, the dmPFC (frontal polar cortex and ACC), the dorsolateral PFC, and the TPJ responded more to social counterfactuals than to private ones. In addition, the dmPFC was more activated during decisions in social contexts than in private contexts. The dissociation we observed in vmPFC patients between private and social counterfactuals is thus consistent with our fMRI results: (1) the mOFC seems critical for encoding and employing regret signals in guiding decisions and (2) by contrast, integrating social comparison signals into the representation of an outcome value might be supported by a more distributed network, as all of our vmPFC patients were sensitive to social comparison.

The normal emotional responses expressed by vmPFC patients during social comparison events might be related to the specificity of our social condition, which participants perceive as competitive. The social impairment typically observed in this patient group might be specific to altruistic choices, whereas competitive situations are spared (Krajbich, Adolphs, Tranel, Denburg, \& Camerer, 2009). Nevertheless, a recent study that included a high number of patients with brain lesion attributed changes in altruistic donation and punishment to the $\mathrm{dmPFC}$, whereas the vmPFC did not bear a robust relationship to social decisions (Moll et al., 2018).

Although social comparison strongly affected how vmPFC patients evaluated the outcomes of their choices, it was less clear how this effect translated into choice behavior. vmPFC patients did not anticipate future regret as much as control participants-especially during early trials-but there was no distinction between private and social trials. Failure to anticipate regret in vmPFC patients is consistent with the proposed role of the vmPFC in generating a mental representation of the choice problem-specifically, integrating the elements relevant to the decision and incorporating information about the potential future consequences of available actions (McCormick, Ciaramelli, De Luca, \& Maguire, 2018).

Interestingly, vmPFC patients progressively avoided regret in their decisions during the course of the task. Such learning effect was not previously reported and could be driven by the social condition, with a spillover effect on private trials. When looking at trial-by-trial effects on future regret avoidance, we observed that vmPFC patients were influenced by past counterfactual information from social trials only, not from private trials. It suggests that emotions elicited by social comparison, such as envy and gloating, are able to drive behavior where private regrets fails as a teaching signal in vmPFC patients. It is also possible that vmPFC patients were able to learn from imitating the choice behavior of their counterparts. Our design does not allow us to disentangle between these two 
possibilities; future research could employ a learning paradigm to investigate this question.

Finally, we were not able to confirm our hypothesis concerning a potential dissociation between ventral and dorsal portions of the medial PFC in their involvement in private and social decisions. Although preliminary evidence associated deficits in anticipating future regret in private settings, no brain regions were associated with deficit in social settings. A larger number of patients with distinct focal vmPFC and dmPFC patients would be needed to conclude on the precise role of these regions in integrating social counterfactual value signals into decisions. In addition, multivariate analyses might be more appropriate to uncover a dissociation between two adjacent brain areas (Mirman et al., 2018; Mah, Husain, Rees, \& Nachev, 2014).

To conclude, our findings suggest that patients with lesions tightly restricted to the vmPFC are unable to reason counterfactually and to anticipate the negative consequences of their choices, while they preserve the ability to experience emotional reactions elicited by social comparison. vmPFC patients were able to learn to anticipate regret over the course of the task, an effect that seems to be driven by their experience in social trials. We suggest that vmPFC patients' deficit in valuation and decisionmaking cannot be attributed to a specific deficit in the processing of social information. The abnormal social behavior that is typically found in this patient group should be attributed to a general decision-making impairment rather than to a failure to take into account other people in evaluating the outcomes of their choices. It remains unclear how their ability to process social comparison signals transfers to future choices. Further work will be needed to confirm that social comparison may serve as a learning signal in patients with lesions in the vmPFC, as it may have great potential for rehabilitation

\section{Acknowledgments}

The authors would like to thank all patients with brain lesion for their participation in the study. We also thank Gabriele Chierchia for his crucial help with recruitment and testing of the Italian control participants, as well as Ben Timberlake for editing the manuscript. The study was funded by the European Research Council (ERC Consolidator Grant 617629) and the Agence National de la Recherche (ANR-11-EMCO-010) grants to G. C. and N. B.

Reprint requests should be sent to Nadège Bault, Center for Mind/Brain Sciences (Cimec), University of Trento, Via Delle Regole 101, 38123 Mattarello, Italy, or via e-mail: Nadege_lab@nbault.net.

\section{REFERENCES}

Abe, H., \& Lee, D. (2011). Distributed coding of actual and hypothetical outcomes in the orbital and dorsolateral prefrontal cortex. Neuron, 70, 731-741.

Appollonio, I., Leone, M., Isella, V., Piamarta, F., Consoli, T., Villa, M. L., et al. (2005). The Frontal Assessment Battery
(FAB): Normative values in an Italian population sample. Neurological Sciences, 26, 108-116.

Bates, E., Wilson, S. M., Saygin, A. P., Dick, F., Sereno, M. I., Knight, R. T., et al. (2003). Voxel-based lesion-symptom mapping. Nature Neuroscience, 6, 448-450.

Bault, N., Coricelli, G., \& Rustichini, A. (2008). Interdependent utilities: How social ranking affects choice behavior. PLoS One, 3, e3477.

Bault, N., Joffily, M., Rustichini, A., \& Coricelli, G. (2011). Medial prefrontal cortex and striatum mediate the influence of social comparison on the decision process. Proceedings of the National Academy of Sciences, U.S.A., 108, 16044-16049.

Bault, N., Wydoodt, P., \& Coricelli, G. (2016). Different attentional patterns for regret and disappointment: An eye-tracking study. Journal of Behavioral Decision Making, 29, 194-205.

Bechara, A., Damasio, H., \& Damasio, A. R. (2000). Emotion, decision making and the orbitofrontal cortex. Cerebral Cortex, 10, 295-307.

Bechara, A., Tranel, D., \& Damasio, H. (2000). Characterization of the decision-making deficit of patients with ventromedial prefrontal cortex lesions. Brain, 123, 2189-2202.

Bechara, A., Tranel, D., Damasio, H., \& Damasio, A. R. (1996). Failure to respond autonomically to anticipated future outcomes following damage to prefrontal cortex. Cerebral Cortex, 6, 215-225.

Beer, J. S., Heerey, E. A., Keltner, D., Scabini, D., \& Knight, R. T. (2003). The regulatory function of self-conscious emotion: insights from patients with orbitofrontal damage. Journal of Personality and Social Psychology, 85, 594-604.

Bell, D. E. (1982). Regret in decision making under uncertainty. Operations Research, 30, 961-981.

Bertossi, E., Aleo, F., Braghittoni, D., \& Ciaramelli, E. (2016). Stuck in the here and now: Construction of fictitious and future experiences following ventromedial prefrontal damage. Neuropsychologia, 81, 107-116.

Boorman, E. D., Behrens, T. E., \& Rushworth, M. F. (2011) Counterfactual choice and learning in a neural network centered on human lateral frontopolar cortex. PLoS Biology, 9, e1001093.

Boorman, E. D., Behrens, T. E., Woolrich, M. W., \& Rushworth, M. F. (2009). How green is the grass on the other side? Frontopolar cortex and the evidence in favor of alternative courses of action. Neuron, 62, 733-743.

Burke, C. J., Tobler, P. N., Baddeley, M., \& Schultz, W. (2010). Neural mechanisms of observational learning. Proceedings of the National Academy of Sciences, U.S.A., 107, 14431-14436.

Camille, N., Coricelli, G., Sallet, J., Pradat-Diehl, P., Duhamel, J. R., \& Sirigu, A. (2004). The involvement of the orbitofrontal cortex in the experience of regret. Science, 304, 1167-1170.

Chang, S. W. C., Gariépy, J.-F., \& Platt, M. L. (2013). Neuronal reference frames for social decisions in primate frontal cortex. Nature Neuroscience, 16, 243-250.

Ciaramelli, E., \& di Pellegrino, G. (2011). Ventromedial prefrontal cortex and the future of morality. Emotion Review, 3, 308-309.

Coricelli, G., Critchley, H. D., Joffily, M., O’Doherty, J. P., Sirigu, A., \& Dolan, R. J. (2005). Regret and its avoidance: A neuroimaging study of choice behavior. Nature Neuroscience, 8, 1255-1262.

Damasio, A. R., Tranel, D., \& Damasio, H. (1990). Individuals with sociopathic behavior caused by frontal damage fail to respond autonomically to social stimuli. Behavioral Brain Research, 41, 81-94.

D'Argembeau, A., Stawarczyk, D., Majerus, S., Collette, F., van der Linden, M., \& Salmon, E. (2010). Modulation of medial prefrontal and inferior parietal cortices when 
thinking about past, present, and future selves. Social Neuroscience, 5, 187-200.

Dubois, B., Slachevsky, A., Litvan, I., \& Pillon, B. (2000). The FAB: A frontal assessment battery at bedside. Neurology, 55, 1621-1626.

Elliott, R., Dolan, R. J., \& Frith, C. D. (2000). Dissociable functions in the medial and lateral orbitofrontal cortex: Evidence from human neuroimaging studies. Cerebral Cortex, 10, 308-317.

Fliessbach, K., Weber, B., Trautner, P., Dohmen, T., Sunde, U., Elger, C. E., et al. (2007). Social comparison affects rewardrelated brain activity in the human ventral striatum. Science, 318, 1305-1308.

Giovagnoli, A. R., Del Pesce, M., Mascheroni, S., Simoncelli, M., Laiacona, M., \& Capitani, E. (1996). Trail making test: Normative values from 287 normal adult controls. Italian Journal of Neurological Sciences, 17, 305-309.

Gu, X., Wang, X., Hula, A., Wang, S., Xu, S., Lohrenz, T. M., et al. (2015). Necessary, yet dissociable contributions of the insular and ventromedial prefrontal cortices to norm adaptation: Computational and lesion evidence in humans. Journal of Neuroscience, 35, 467-473.

Haroush, K., \& Williams, Z. M. (2015). Neuronal prediction of opponent's behavior during cooperative social interchange in primates. Cell, 160, 1233-1245.

Hayden, B. Y., Pearson, J. M., \& Platt, M. L. (2009). Fictive reward signals in the anterior cingulate cortex. Science, 324, 948-950

Kim, K.-U., Huh, N., Jang, Y., Lee, D., \& Jung, M. W. (2015). Effects of fictive reward on rat's choice behavior. Scientific Reports, 5, 840.

Koenigs, M., \& Tranel, D. (2007). Irrational economic decisionmaking after ventromedial prefrontal damage: Evidence from the ultimatum game. Journal of Neuroscience, 27, 951-956.

Koenigs, M., Young, L., Adolphs, R., Tranel, D., Cushman, F., Hauser, M., et al. (2007). Damage to the prefrontal cortex increases utilitarian moral judgements. Nature, 446, 908-911.

Krajbich, I., Adolphs, R., Tranel, D., Denburg, N. L., \& Camerer, C. F. (2009). Economic games quantify diminished sense of guilt in patients with damage to the prefrontal cortex. Journal of Neuroscience, 29, 2188-2192.

Kringelbach, M. L., \& Rolls, E. T. (2004). The functional neuroanatomy of the human orbitofrontal cortex: Evidence from neuroimaging and neuropsychology. Progress in Neurobiology, 72, 341-372.

Levens, S. M., Larsen, J. T., Bruss, J., Tranel, D., Bechara, A., \& Mellers, B. A. (2014). What might have been? The role of the ventromedial prefrontal cortex and lateral orbitofrontal cortex in counterfactual emotions and choice. Neuropsychologia, 54, 77-86.

Lohrenz, T., McCabe, K., Camerer, C. F., \& Montague, P. R. (2007). Neural signature of fictive learning signals in a sequential investment task. Proceedings of the National Academy of Sciences, U.S.A., 104, 9493-9498.

Loomes, G., \& Sugden, R. (1982). Regret theory: An alternative theory of rational choice under uncertainty. Economic Journal, 92, 805-824.

Mah, Y.-H., Husain, M., Rees, G., \& Nachev, P. (2014). Human brain lesion-deficit inference remapped. Brain, 137, 2522-2531.

McCormick, C., Ciaramelli, E., De Luca, F., \& Maguire, E. A. (2018). Comparing and contrasting the cognitive effects of hippocampal and ventromedial prefrontal cortex damage: A review of human lesion studies. Neuroscience, 374 , 295-318.

Mirman, D., Landrigan, J.-F., Kokolis, S., Verillo, S., Ferrara, C., \& Pustina, D. (2018). Corrections for multiple comparisons in voxel-based lesion-symptom mapping. Neuropsychologia, $115,112-123$.

Mitchell, J. P., Macrae, C. N., \& Banaji, M. R. (2006). Dissociable medial prefrontal contributions to judgments of similar and dissimilar others. Neuron, 50, 655-663.

Moll, J., de Oliveira-Souza, R., Basilio, R., Bramati, I. E., Gordon, B., Rodríguez-Nieto, G., et al. (2018). Altruistic decisions following penetrating traumatic brain injury. Brain, 141, 1558-1569.

Moretti, L., Dragone, D., \& di Pellegrino, G. (2009). Reward and social valuation deficits following ventromedial prefrontal damage. Journal of Cognitive Neuroscience, 21, 128-140.

Nelson, H. E. (1976). A modified card sorting test sensitive to frontal lobe defects. Cortex, 12, 313-324.

Orthony, A., Clore, G. L., \& Collins, A. (1988). The cognitive structure of emotions. Cambridge: Cambridge University Press.

Padoa-Schioppa, C., \& Assad, J. A. (2006). Neurons in the orbitofrontal cortex encode economic value. Nature, 441, 223-226.

Rolls, E. T., Hornak, J., Wade, D., \& McGrath, J. (1994). Emotion-related learning in patients with social and emotional changes associated with frontal lobe damage. Journal of Neurology, Neurosurgery, and Psychiatry, 57, 1518-1524.

Rorden, C., Karnath, H.-O., \& Bonilha, L. (2007). Improving lesion-symptom mapping. Journal of Cognitive Neuroscience, 19, 1081-1088.

Rudebeck, P. H., Bannerman, D. M., \& Rushworth, M. F. (2008). The contribution of distinct subregions of the ventromedial frontal cortex to emotion, social behavior, and decision making. Cognitive, Affective \& Behavioral Neuroscience, 8 , 485-497.

Shamay-Tsoory, S. G., Tibi-Elhanany, Y., \& Aharon-Peretz, J. (2007). The green-eyed monster and malicious joy: The neuroanatomical bases of envy and gloating (schadenfreude). Brain, 130, 1663-1678.

Steiner, A. P., \& Redish, A. D. (2014). Behavioral and neurophysiological correlates of regret in rat decision-making on a neuroeconomic task. Nature Neuroscience, 17, 995-1002.

Stuss, D. T., \& Benson, D. F. (1984). Neuropsychological studies of the frontal lobes. Psychological Bulletin, 95, 3-28.

Sul, S., Tobler, P. N., Hein, G., Leiberg, S., Jung, D., Fehr, E., et al. (2015). Spatial gradient in value representation along the medial prefrontal cortex reflects individual differences in prosociality. Proceedings of the National Academy of Sciences, U.S.A., 112, 7851-7856.

Tombaugh, T. N. (2004). Trail making test A and B: Normative data stratified by age and education. Archives of Clinical Neuropsychology, 19, 203-214.

Wheeler, E. Z., \& Fellows, L. K. (2008). The human ventromedial frontal lobe is critical for learning from negative feedback. Brain, 131, 1323-1331.

Zeelenberg, M., van Dijk, W. W., Manstead, A. S. R., \& van der Pligt, J. (2000). On bad decisions and disconfirmed expectancies: The psychology of regret and disappointment. Cognition and Emotion, 14, 521-541. 